\title{
LBM-EP: Lattice-Boltzmann Method for Fast Cardiac Electrophysiology Simulation from 3D Images
}

\author{
S. Rapaka ${ }^{1}$, T. Mansi ${ }^{1}$, B. Georgescu ${ }^{1}$, M. Pop ${ }^{2}$, G.A. Wright ${ }^{2}$, \\ A. Kamen ${ }^{1}$, and Dorin Comaniciu ${ }^{1}$ \\ 1 Siemens Corporation, Corporate Research and Technology, \\ Imaging and Computer Vision, Princeton, NJ, USA \\ 2 Department of Medical Biophysics, University of Toronto, \\ Sunnybrook Health Sciences Centre, Imaging Research, Toronto, ON, Canada
}

\begin{abstract}
Current treatments of heart rhythm troubles require careful planning and guidance for optimal outcomes. Computational models of cardiac electrophysiology are being proposed for therapy planning but current approaches are either too simplified or too computationally intensive for patient-specific simulations in clinical practice. This paper presents a novel approach, LBM-EP, to solve any type of mono-domain cardiac electrophysiology models at near real-time that is especially tailored for patient-specific simulations. The domain is discretized on a Cartesian grid with a level-set representation of patient's heart geometry, previously estimated from images automatically. The cell model is calculated node-wise, while the transmembrane potential is diffused using Lattice-Boltzmann method within the domain defined by the levelset. Experiments on synthetic cases, on a data set from CESC'10 and on one patient with myocardium scar showed that LBM-EP provides results comparable to an FEM implementation, while being 10 - 45 times faster. Fast, accurate, scalable and requiring no specific meshing, LBM-EP paves the way to efficient and detailed models of cardiac electrophysiology for therapy planning.
\end{abstract}

\section{Introduction}

Since the seminal work of Hodgkin and Huxley [5, a large variety of models have been proposed to simulate the propagation of the action potential (AP) across the heart muscle, with various degrees of complexity ([3]). Biophysical models aim to capture the ion interactions and protein mechanisms that regulate the AP. At a higher scale, phenomenological models have been developed to mimic the AP without directly considering the underlying molecular mechanisms. Finally, Eikonal models do not simulate the AP altogether but the propagation of the electrical front directly. Since these models are generative, they may constitute efficient tools for therapy planning. Phenomenological models provide a good compromise between model details, being able to capture most of the pathological conditions, and complexity. Recent works demonstrated that those models 
can be personalised from clinical data 1 . However, because they solve stiff partial differential equations (PDE), they are still too computationally demanding for day-to-day clinical setups and intervention guidance. Another limitation is the requirement of high-quality/high-resolution volume meshes, which can be difficult to obtain from patient-specific anatomies.

In recent years, the lattice-Boltzmann method (LBM) 2] has developed as a powerful technique for accurate simulation of a large class of partial-differential equations. In particular, it has been successfully applied to pattern-forming reaction-diffusion equations $([4)$. While originally developed from cellular automata models of fluid flows, the method has found a firm theoretical basis in kinetic theory of weakly-compressible fluid flows. Some of the key strengths of this method are, $i$ ) local nature of the computational algorithm, which provides very high scalability on modern parallel computing architectures, ii) secondorder accuracy in space, and iii) simplicity of implementation on a uniform Cartesian grid.

Motivated by the recent breakthrough in LBM, we present a novel framework, henceforth called LBM-EP, for efficient patient-specific simulations of cardiac electrophysiology models at near real-time. Although general, the method is illustrated in this study on the Mitchell-Schaeffer model [6]. Sec. 2 presents a description of the algorithms used to process the medical images, and the lattice-Boltzmann algorithm used for propagating the action-potential. Sec. 3 compares the simulation results computed with the proposed LBM-EP with an FEM implementation of Mitchell-Schaeffer model in synthetic scenarios, a dataset of CESC'10 Grand Challenge and one patient with a myocardium scar, showing an accuracy in the range of the variability reported in the literature and a speed-up of about $10-45 \times$ with respect to FEM. Sec. 4 concludes the paper.

\section{Methods}

\subsection{Computational Domain Preparation from Medical Images}

LBM-EP being solved on Cartesian grids (Sec. 2.3), its application to clinical images is relatively immediate. Starting from a cardiac image (e.g. cine MRI), the left endocardium, right endocardium and epicardium are automatically segmented using a machine learning approach [11] and fused in one surface representing the myocardium while preserving their anatomical label. A level-set representation of that surface is then computed on an isotropic Cartesian grid. Based on the labels, grid nodes lying at the heart endocardia are marked for electrophysiology stimulation and synthetic fibers are computed by linearly interpolating the elevation angle from $-70^{\circ}$ at the epicardium to $+70^{\circ}$ at the endocardium [3]. Scars can be reported in the domain through level set (Sec. 3.3).

\subsection{Mitchell-Schaeffer Model of Action Potential}

Mitchell-Schaeffer (M-S) model [6] is employed here although the method can be extended to other mono-domain models. The model (Eq. (1)) relates the 
normalized transmembrane $\operatorname{AP} v(t) \in[0,1]$ to an inward gated current $J_{\text {in }}=$ $h v^{2}(1-v) / \tau_{i n}$, which captures the fast acting currents using the gating variable $h(t)$, and an outward un-gated current $J_{\text {out }}=-v / \tau_{\text {out }}$, which accounts for transmembrane voltage decrease. A transient stimulus current $J_{\text {stim }}$ is added to the model to simulate electrical pacing.

$$
\frac{\partial v}{\partial t}=J_{\text {int }}+J_{\text {out }}+J_{\text {stim }}+c \nabla \cdot D \nabla v
$$

The gating variable $h(t)$ evolves according to $\frac{d h}{d t}=\frac{1-h}{\tau_{\text {open }}}$ if $v<v_{\text {gate }}$, otherwise $\frac{d h}{d t}=\frac{-h}{\tau_{\text {close }}}$ where $v_{\text {gate }}$ is the change-over voltage, $D=\rho \mathrm{Id}+(1-\rho) \mathbf{a a}^{\top}$ is the anisotropic diffusion tensor along the fiber direction a with anisotropy ratio $\rho, c$ is the diffusion coefficient along the fibers. $\tau_{\text {in }}, \tau_{\text {out }}, \tau_{\text {open }}$ and $\tau_{\text {close }}$ are directly related to the shape and duration of the AP $([\underline{6})$, which makes their calibration possible from clinical data.

\subsection{Lattice-Boltzmann Model of Cardiac Electrophysiology}

Eq. (11) is solved on the Cartesian grid (Sec. 2.1) using Lattice-Boltzmann method with a 7 -connectivity topology (6 connections + central position) and Neumann boundary conditions. The gating variable $h(t)$ is updated at every node of the grid using a forward Euler scheme. The fundamental variable of LBM is the vector of distribution functions $\mathbf{f}(\mathbf{x})=\left\{f_{i}(\mathbf{x})\right\}_{i=1 \ldots 7}$, where $f_{i}(\mathbf{x})$ represents the probability of finding a particle travelling along the edge $\mathbf{e}_{i}$ of node $\mathbf{x}$. The governing equation at $\mathbf{x}$ for the edge $\mathbf{e}_{i}$ is composed of two successive steps:

$$
\begin{aligned}
& f_{i}^{*}=f_{i}-A_{i j}\left(f_{j}-\omega_{j} v\right)+\delta t \omega_{i}\left(J_{\text {in }}+J_{\text {out }}+J_{\text {stim }}\right), \\
& f_{i}\left(\mathbf{x}+\mathbf{e}_{i}, t+\delta t\right)=f_{i}^{*}(\mathbf{x}, t)
\end{aligned}
$$

where, the collision matrix $\mathrm{A}=\left(A_{i j}\right)_{i, j \in \llbracket 1,7 \rrbracket}$ relaxes the distribution function $f_{i}$ towards the local value of the potential, $v, f_{i}^{*}$ is an intermediate, post-collision state of the distribution function, and $\omega_{i}$ is a weighting factor that depends on lattice connectivity, here $\omega_{i}=1 / 8$ for the edges to the six neighbors and $\omega_{i}=1 / 4$ for the central position. The transmembrane AP is related to the $f_{i}$ 's through $v(\mathbf{x}, t)=\sum_{i} f_{i}(\mathbf{x}, t)$. For each time step $\delta t$, a strictly local collision rule (Eq. (2) ) is applied to the distribution functions at each node. Post-collision, the distribution functions stream along their corresponding edges to the neighboring nodes (Eq. (3) ). In its simplest form, the collision matrix is diagonal with a characteristic relaxation time $\tau, \mathrm{A}=(1 / \tau) \mathrm{I}$, where $\mathrm{I}$ is the $7 \times 7$ identity matrix.

At the problem boundaries, the streaming step requires the specification of additional incoming distribution functions to ensure proper boundary conditions. It can be shown that the potential gradient at a node is related to the $f_{i}$ 's through $c \nabla v=(1-1 / 2 \tau) \sum_{i} f_{i} \mathbf{e}_{i}$ [4]. The Neumann boundary condition for potential on a surface simplifies to $\sum_{i} f_{i} \mathbf{e}_{i} \cdot \mathbf{n}=0$. If the boundary is normal to any edge of the lattice, the Neumann boundary condition is automatically recovered if the incoming distribution at the node is equal to the outgoing one. Complex geometries can be handled easily by means of a level set formulation. The incoming 
distribution is calculated automatically from the distance to the wall at nodes close to the boundary as provided by the level-set [10], thus enabling simulations in complex domain without requiring advanced meshing algorithms.

Remarkably, this simple model can be shown to reproduce the reaction-diffusion equation Eq. (1) with an isotropic diffusion coefficient of $c=(2 \tau-1) / 8$ (see [4] for the proof). To extend the model for anisotropic diffusion, like in the heart, the matrix $\mathrm{A}$ is replaced by $\mathrm{A}=\mathrm{M}^{-1} \mathrm{SM}$ [9], where

$$
M=\left(\begin{array}{ccccccc}
1 & 1 & 1 & 1 & 1 & 1 & 1 \\
1 & -1 & 0 & 0 & 0 & 0 & 0 \\
0 & 0 & 1 & -1 & 0 & 0 & 0 \\
0 & 0 & 0 & 0 & 1 & -1 & 0 \\
1 & 1 & 1 & 1 & 1 & 1 & -6 \\
1 & 1 & -1 & -1 & 0 & 0 & 0 \\
1 & 1 & 1 & 1 & -2 & -2 & 0
\end{array}\right) S^{-1}=\left(\begin{array}{ccccccc}
\tau_{1} & 0 & 0 & 0 & 0 & 0 & 0 \\
0 & \tau_{11} & \tau_{12} & \tau_{13} & 0 & 0 & 0 \\
0 & \tau_{21} & \tau_{22} & \tau_{23} & 0 & 0 & 0 \\
0 & \tau_{31} & \tau_{32} & \tau_{33} & 0 & 0 & 0 \\
0 & 0 & 0 & 0 & \tau_{5} & 0 & 0 \\
0 & 0 & 0 & 0 & 0 & \tau_{6} & 0 \\
0 & 0 & 0 & 0 & 0 & 0 & \tau_{7}
\end{array}\right)
$$

The first row of $\mathrm{M}$ corresponds to $v=\sum_{i} f_{i}$, while rows $2-4$ are the three components of the potential gradient. The relaxation times $\left(\tau_{i j}\right)_{i, j \in \llbracket 1,3 \rrbracket}$ are related to the components of the diffusion tensor through $\tau_{i j}=\delta_{i j} / 2+4 D_{i j} \delta t / \delta x^{2}$. The relaxation times $\tau_{1}$ related to the potential and $\tau_{5}, \tau_{6}$ and $\tau_{7}$ related to the higher order moments do not directly effect the diffusion solution, but effect the stability of the method. In this work, we use $\tau_{1}=1$ and $\tau_{5}=\tau_{6}=\tau_{7}=1.33$. Algo. 1 summarizes the main steps of the method.

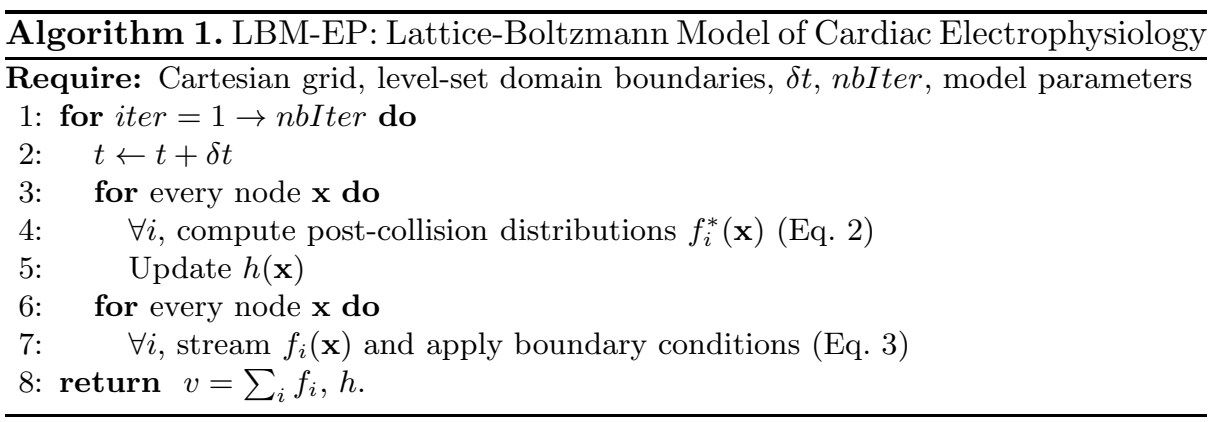

\section{Experiments and Results}

All experiments were executed on a standard Windows XP desktop machine (Intel Xeon, 2.40GHz octo-core, 4GB RAM). LBM-EP was implemented in Fortran with no particular optimization. A semi-implicit, anisotropic finite element implementation of M-S model, called FEM-EP, was used for comparisons. FEM-EP was based on linear tetrahedra and parallel optimization (OpenMP).

\subsection{Quantitative Evaluation on Synthetic Scenarios}

We first compared the performance of LBM-EP with respect to FEM-EP in two different scenarios representing the main pathological features. To that end, a $10 \times 10 \times 0.5 \mathrm{~cm}$ slab was discretized into $401 \times 401 \times 3$ nodes $(1,920,000$ tetrahedra for FEM-EP). For both LBM-EP and FEM-EP, we used $\delta t=0.1 \mathrm{~ms}$, $\tau_{\text {close }}=150 \mathrm{~ms}, \tau_{\text {open }}=120 \mathrm{~ms}, \tau_{\text {in }}=0.3 \mathrm{~ms}, \tau_{\text {out }}=6 \mathrm{~ms}, v_{\text {gate }}=0.13$, 
$c=0.0003 \mathrm{~cm}^{2} / \mathrm{ms}$ and $\rho=1$ (isotropic diffusion) [6]. An electrical stimulation was applied at the nodes $(x, y=0, z)$ for $1 \mathrm{~ms}$ duration by setting $v=1$ at these nodes. Grid resolution analysis (not reported here) showed that numerical convergence was reached for both models at that spatial and temporal resolution.

We first tested the ability of LBM-EP to capture front-bending around a scar. A scar region was simulated within the domain (Fig. 11 left panels) by: setting the diffusion coefficient $c=0$ for FEM-EP, defining Neumann boundary conditions for LBM-EP. From the computed depolarization times (Fig. 1, left panels), one can see that both models yielded very similar behavior. The AP front calculated by LBM-EP correctly rotated around the scar. Where the front was not perturbed by the scar, both models yielded nearly identical results, as quantified by the AP at Point $2(0.3 \mathrm{~ms}$ difference in depolarization time, Fig. 11 right panel). Near the scar, slight differences could be identified, which resulted in a difference of $9.7 \mathrm{~ms}$ in depolarization time at Point 1 . However, this cannot be interpreted as a limitation of LBM-EP, which captured perfectly the Neumann boundary conditions around the scar, contrary to FEM-EP.
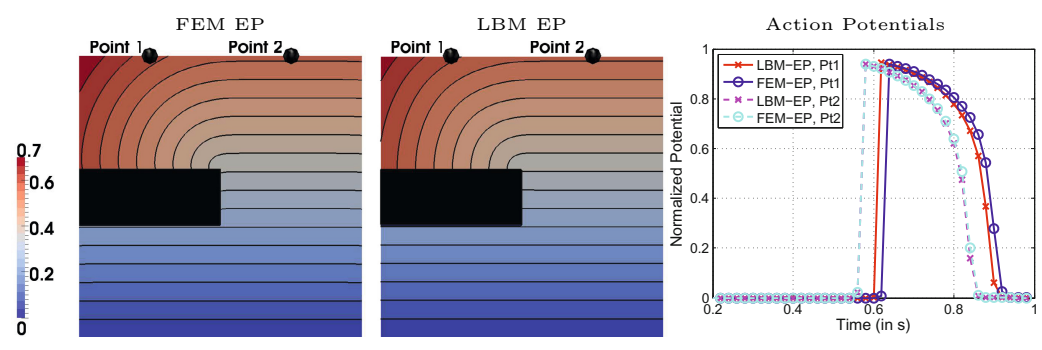

Fig. 1. Left panels: Computed depolarization times (in $m s$ ) in a homogeneous medium with scar (in black). LBM-EP captured front rotation around the scar. Right panel: action potential at points 1 and 2, showing consistent results between both models.

We then tested the ability of LBM-EP to simulate vortex formation due to premature stimulation. For that, we set the diffusion coefficient to $0.0012 \mathrm{~cm}^{2} / \mathrm{ms}$, removed the scar and applied a second stimulation at the nodes $(x \leq 0.5, y=$ $0.5, z)$ at $t=452 \mathrm{~ms}$ for both methods. As shown in Fig. 2] the patterns obtained with LBM-EP were similar to those obtained with FEM-EP. LBM-EP could be used to simulate complex pathologies like fibrillation or tachycardia.

Computation Time. For all experiments, FEM-EP required $\approx 700 \mathrm{~ms}$ per iteration whereas LBM-EP required only $\approx 80 \mathrm{~ms}$, about $8.75 \times$ speed-up.

\subsection{Comparison with Published Results on CESC'10 Data}

We evaluated LBM-EP performance in a dataset distributed during CESC'10 MICCAI Grand Challenge with respect to FEM-EP and to a recently published benchmark [1]. Our purpose being evaluation and not personalization, we did not adjust the parameters locally. We thus compared our results with the generic benchmark of the ionic ten Tusscher-Panfilov model only [1. CESC'10 dataset 


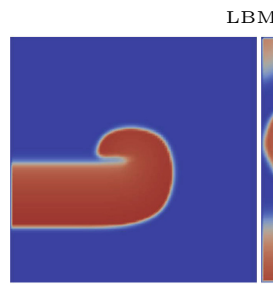

$610 \mathrm{~ms}$

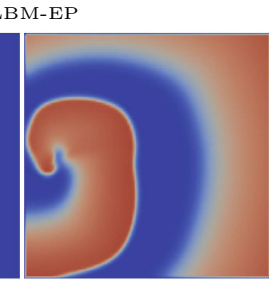

$1320 \mathrm{~ms}$

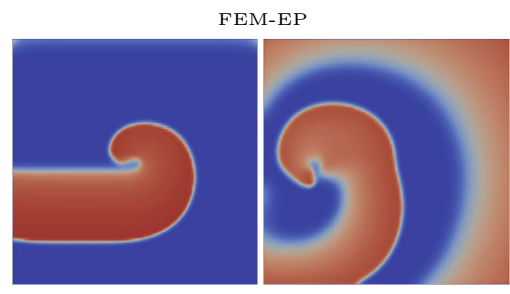

$610 \mathrm{~ms}$

$1320 \mathrm{~ms}$

Fig. 2. LBM-EP succesfully captured vortex formation due to premature stimulation

consisted in an explanted porcine heart, and comprised optical fluorescence images of transmembrane potential and high-resolution diffusion-weighted (DW) MRI images ([7]). The optical images contained the depolarization and repolarization phase of transmembrane potential under left endocardium and right epicardium pacings at $1.1 \mathrm{~Hz}$. Finally, the mesh constructed from DW-MRI had the fiber directions integrated, providing a complete model of the heart anatomy.

LBM-EP was computed on $0.5 \mathrm{~mm}$ grid while FEM-EP was computed on the provided tetrahedra mesh with $0.5 \mathrm{~mm}$ average edge-length. The time steps were set to $\delta t_{L B M-E P}=0.1 \mathrm{~ms}$ and $\delta t_{F E M-E P}=0.5 \mathrm{~ms}$. Myocardium fibers defined on the tetrahedra mesh were rasterized on the LBM lattice for simulation. The generic M-S parameters were used for both models [6, with a diffusion coefficient $c=0.0035 \mathrm{~cm}^{2} / \mathrm{ms}$ and anisotropy ratio $\rho=0.25$. As it can be seen from Fig. 3, LBM-EP simulation was qualitatively similar to FEM-EP for both pacing conditions in terms of depolarization isochrone patterns. The difference in absolute depolarization time was mostly due to the different computational domain, in particular regarding the precise location of the excitation nodes and the fiber orientation, which was locally altered by the rasterization. Compared to the CESC'10 benchmark, LBM-EP provided similar depolarization patterns, suggesting promising reproducibility and validity. Finally, while FEM-EP required $\approx 16 s$ per iteration, LBM-EP took only $\approx 0.35 s$ per iteration, which corresponds to a speed-up of $45 \times$. It should be noted that the computational efficiency of our FEM implementation was similar to those reported in the literature, $\approx 1 \mathrm{~s} /$ iteration on the $1.5 \mathrm{~mm}$ mesh provided by the challengers as in 8 .

\subsection{Real Case Example}

We finally illustrate how LBM-EP can be used in a real clinical scenario on a patient with myocardium scar due to previous surgery. Parameters were kept generic as no electrophysiology data were available. Fast conductivity was modeled on the endocardium to mimic the Purkinje fibers. The scar was represented as a level-set to ensure Neumann boundary conditions. Fig. 4 shows the depolarization time isochrones, illustrating some delays at the apex due to the scar. Elsewhere in the myocardium the isochrones presented with patterns similar to what has been reported in the literature [3. For this patient, one time step was calculated in $0.2 \mathrm{~s}$ for a grid size of $1 \mathrm{~mm}$. 


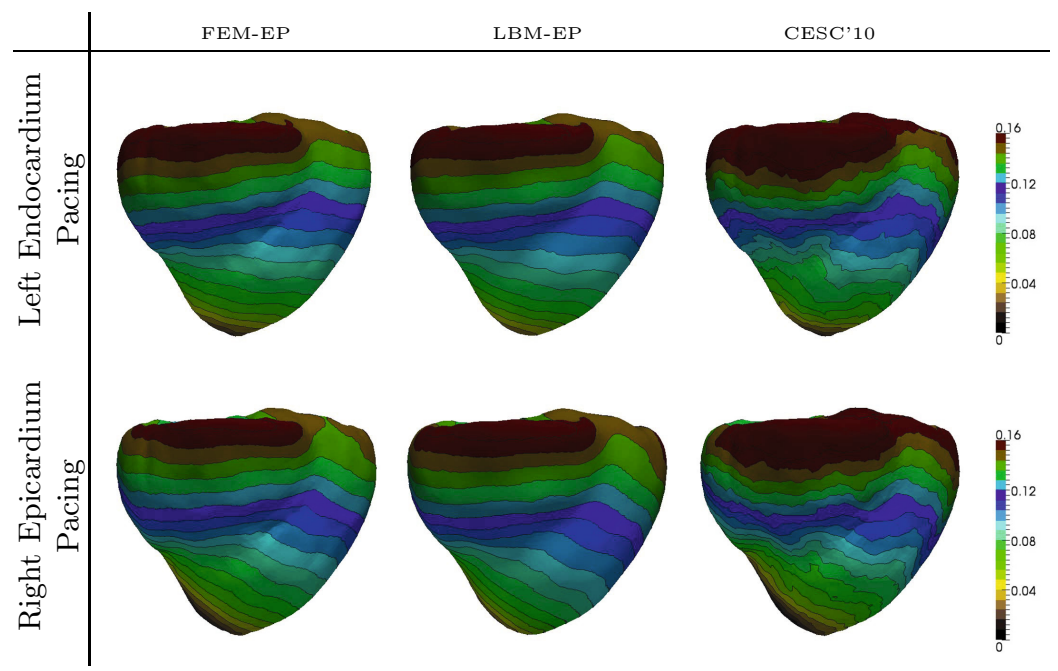

Fig. 3. FEM-EP and LBM-EP simulations using Mitchell-Schaeffer model and CESC'10 data using ten Tusscher-Panfilov model (see text for details)

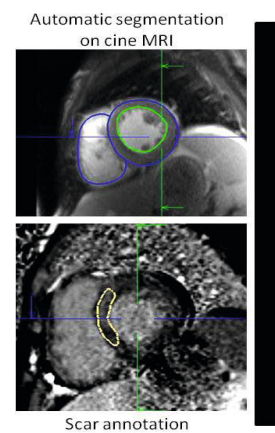

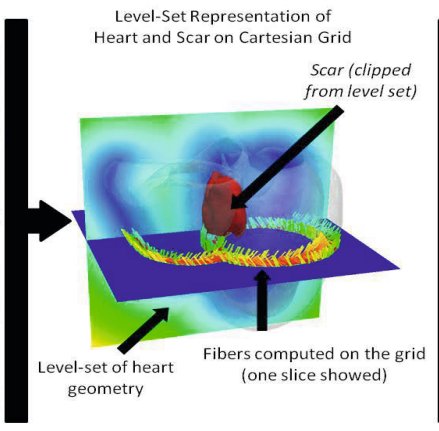

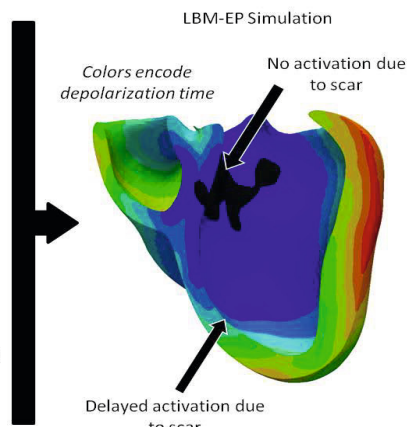

to scar

Fig. 4. LBM-EP simulation on one patient with myocardium scar

\section{Conclusion and Future Work}

We have presented in this paper, to the best of our knowledge, the first application of a near-real time lattice-Boltzmann model for general monodomain models of cardiac electrophysiology. Node-based by construction, our framework does not require advanced meshing and can be applied directly from images by means of level-sets. Through comparisons with the traditional finite-element method, we have empirically shown the applicability of LBM-EP to cardiac electrophysiology. A comprehensive description of the theory along with detailed studies of convergence and accuracy are being prepared as a larger contribution. LBM-EP provides second order accuracy in space, can be easily extended to any type of mono-domain cellular model, and, above all, offers between $10-45 \times$ speed-up with respect to traditional FEM. Preliminary experiments on graphi- 
cal processing units (GPUs) showed the potential for another order-of-magnitude improvement in computational speed. This is the first time, to the best of our knowledge, that very fast simulations of cardiac electrophysiology is achieved with a detailed model. Our method may thus constitute an ideal framework for patient-specific simulations for therapy planning and real-time guidance.

Acknowledgement. We would like to thank Prof. Oscar Camara of PhySense group at Information and Communication Technologies Department (DTIC) at Universitat Pompeu Fabra, Barcelona, Spain for providing the CESC'10 benchmark results.

\section{References}

1. Camara, O., Sermesant, M., Lamata, P., Wang, L., Pop, M., Relan, J., De Craene, M., Delingette, H., Liu, H., Niederer, S., Pashaei, A., Plank, G., Romero, D., Sebastian, R., Wong, K.C., Zhang, H., Ayache, N., Frangi, A., Shi, P., Smith, N., Wright, G.: Inter-Model Consistency and Complementarity: Learning from exvivo Imaging and Electrophysiological Data towards an Integrated Understanding of Cardiac Physiology. Prog. Biophys. Mol. Biol. 107, 122-133 (2011)

2. Chen, S., Doolen, G.D.: Lattice Boltzmann method for fluid flows. Annu. Rev. Fluid Mech. 30(1), 329-364 (1998)

3. Clayton, R.H., Bernus, O., Cherry, E.M., Dierckx, H., Fenton, F.H., Mirabella, L., Panfilov, A.V., Sachse, F.B., Seemann, G., Zhang, H.: Models of cardiac tissue electrophysiology: Progress, challenges and open questions. Prog. Biophys. Mol. Biol. 104(1-3), 22 (2011)

4. Dawson, S.P., Chen, S., Doolen, G.D.: Lattice Boltzmann computations for reaction-diffusion equations. J. Chem. Phys. 98(2), 1514-1523 (1993)

5. Hodgkin, A.L., Huxley, A.F.: A quantitative description of ion currents and its applications to conduction and excitation in nerve membranes. J. Physiol. 117(4), 500-544 (1952)

6. Mitchell, C.C., Schaeffer, D.G.: A two-current model for the dynamics of cardiac membrane. Bull. Math. Biol. 65(5), 767-793 (2003)

7. Pop, M., Sermesant, M., Lepiller, D., Truong, M.V., McVeigh, E.R., Crystal, E., Dick, A., Delingette, H., Ayache, N., Wright, G.A.: Fusion of optical imaging and mri for the evaluation and adjustment of macroscopic models of cardiac electrophysiology: A feasibility study. Med. Image Anal. 13(2), 370-380 (2009)

8. Relan, J., Sermesant, M., Pop, M., Delingette, H., Sorine, M., Wright, G., Ayache, N.: Volumetric Prediction of Cardiac Electrophysiology using a Heart Model Personalised to Surface Data. In: CI2BM 2009 - MICCAI Workshop on Cardiovascular Interventional Imaging and Biophysical Modelling (2009)

9. Yoshida, H., Nagaoka, M.: Multiple-relaxation-time lattice Boltzmann model for the convection and anisotropic diffusion equation. J. Comp. Phys. 229, 7774-7795 (2010)

10. Yu, D., Mei, R., Luo, L., Shyy, W.: Viscous flow computations with the method of lattice Boltzmann equation. Prog. Aero. Sci. 39(5), 329-367 (2003)

11. Zheng, Y., Barbu, A., Georgescu, B., Scheuering, M., Comaniciu, D.: Four-chamber heart modeling and automatic segmentation for 3-D cardiac CT volumes using marginal space learning and steerable features. IEEE Trans. Med. Imaging 27(11), 1668-1681 (2008) 